\title{
Implicit Weighted Essentially Nonoscillatory Schemes for the Compressible Navier-Stokes Equations
}

\author{
Jaw-Yen Yang* \\ National Taiwan University, Taipei 10764, Taiwan, Republic of China \\ Yeu-Ching Perng ${ }^{\dagger}$ \\ Chung-Shan Institute of Science and Technology, Taoyuan 33509, Taiwan, Republic of China \\ and \\ Ruey-Hor Yen ${ }^{\ddagger}$ \\ National Taiwan University, Taipei 10764, Taiwan, Republic of China
}

\begin{abstract}
A class of lower-upper symmetric Gauss-Seidel implicit weighted essentially nonoscillatory (ENO) schemes for solving the two- and three-dimensional compressible Navier-Stokes equations with pointwise version of BaldwinBarth one-equation turbulence model is presented (Baldwin, B. S., and Barth, T. J., "A One-Equation Turbulence Transport Model for High Reynolds Number Wall Bounded Flows,” AIAA Paper 91-0610, 1991). A weighted ENO (WENO) spatial operator is employed for inviscid fluxes and central differencing for viscous fluxes. A numerical flux of the WENO scheme in flux limiter form is adopted, which consists of first-order and high-order fluxes and allows for a more flexible choice of first-order dissipative methods. The computations are performed for the two-dimensional turbulent flows over NACA 0012 and Royal Aircraft Establishment 2822 airfoil and the threedimensional turbulent flow over an ONERA M6 wing. The present solutions are compared with experimental data and other computational results and exhibit good agreement.
\end{abstract}

\section{Introduction}

$\mathbf{T}$ HE essentially nonoscillatory (ENO) schemes developed by Harten et al. ${ }^{1}$ are uniformly high-order accurate right up to discontinuities, while keeping a sharp, ENO shock transition. Later, Shu and Osher ${ }^{2,3}$ devised an efficient flux version. Since then, ENO schemes have been successfully applied to many different fields as noted in Ref. 4. However, they also have certain drawbacks. One problem is that the convergence rate for the implicit ENO scheme is generally poor. However implicit total variation diminishing (TVD) schemes ${ }^{5}$ as constructed out of Harten's TVD scheme ${ }^{6}$ can achieve good convergence. Another problem is that an ENO scheme is not effective on vector supercomputers due to its heavy use of logical statements.

Rogerson and Meiburg ${ }^{7}$ studied the convergence properties of ENO schemes, and they found that the numerical solution of ENO schemes does not converge uniformly. $\mathrm{Shu}^{8}$ proposed a modified ENO scheme, which recovers the correct order of accuracy for the test problem. A comparison of finite volume and finite difference implementation of high-order accurate ENO schemes was given by Casper et al. ${ }^{9}$

The weighted ENO (WENO) schemes proposed recently by Liu et al. ${ }^{10}$ and extended by Jiang and Shu ${ }^{11}$ can overcome these drawbacks while keeping the robustness and high-orderaccuracy of ENO schemes. The primary concept of WENO schemes is that, instead of using only one of the candidate stencils based on divided difference to form the reconstruction, one uses a convex combination of all of the candidate stencils. Each of the candidate stencils is assigned a weight that determines the appropriate contribution of this stencil to the final approximation of the numerical flux. Atkins ${ }^{12}$ also devised a version of ENO schemes using a different weighted average of stencils. A class of implicit WENO schemes has been successfully applied to incompressible flow problems by Chen et al. ${ }^{13}$ and Yang et al. ${ }^{14}$ based on Chorin's ${ }^{15}$ artificial compressibility formulation. Good convergencerate to a steady-statesolution has been illustrated.

Received 4 October 1999; revision received 19 May 2000; accepted for publication 22 March 2001. Copyright (C) 2001 by the American Institute of Aeronautics and Astronautics, Inc. All rights reserved.

*Professor, Institute of Applied Mechanics, Department of Mechanical Engineering; yangjy@ spring. iam.ntu.edu.tw. Member AIAA.

Research Scientist.

¥Professor, Department of Mechanical Engineering.
In this paper, following Chen et al. ${ }^{13}$ and Yang et al..${ }^{14}$ an implicit version of the WENO scheme (see Ref. 11) is adopted for the twoand three-dimensional compressible Navier-Stokes equations for computing steady-state flows. A numerical flux of WENO scheme in flux limiter form ${ }^{16}$ is presented that consists of first-order and high-order fluxes and allows for a more flexible choice of first-order dissipative entropy satisfying methods. Many first-order dissipative schemes can be used. Here, we employ the Roe scheme ${ }^{17}$ (with Harten's entropy $\mathrm{fix}^{6}$ ) as the basic first-order dissipative methods.

For turbulent flow calculations, a pointwise version of the Baldwin-Barth one-equation turbulence mode ${ }^{18}$ modified by Goldberg and Ramakrishnan ${ }^{19}$ is adopted, which is based on the $\kappa-\varepsilon$ two-equation model. This model consists entirely of pointwise terms, that is, no term involves wall distance explicitly. Consequently, the resulting model provides a desirable tool for numerical computation of flow involving complex geometry. The performance of this model has been tested through comparison with experimental data of several well-documented flow cases, covering both wallbounded and free shear flows. ${ }^{19}$

To improve the efficiency and convergence to steady state, the lower-upper symmetric Gauss-Seidel (LU-SGS) implicit algorithm (see Ref. 20) is adopted. It has been demonstrated by Yoon and $\mathrm{Kwak}^{21-23}$ that the LU-SGS scheme requires less CPU time per iteration than most existing time marching methods on Cray supercomputers. The LU-SGS scheme is not only unconditionally stable but also completely vectorizable in any dimensions. We apply the resulting schemes to compute standard transonic flows over NACA 0012 and Royal Aircraft Establishment (RAE) 2822 airfoils and three-dimensional transonic flow over ONERA M6 wing to test both the convergence rate and the accuracy of the methods.

\section{Governing Equations}

The governing equations are the unsteady, mass-averaged, compressible Navier-Stokes equations, which express the conservation of mass, momentum, and energy for a viscous gas. The pointwise version of the Baldwin-Barth one-equation turbulence mode ${ }^{18}$ as devised in Ref. 19 is adopted. In the Cartesian coordinates, the threedimensional governing equations are given by

$$
\frac{\partial Q}{\partial t}+\frac{\partial E}{\partial x}+\frac{\partial F}{\partial y}+\frac{\partial G}{\partial z}=\frac{\partial E_{v}}{\partial x}+\frac{\partial F_{v}}{\partial y}+\frac{\partial G_{v}}{\partial z}+H
$$


where

$$
\begin{gathered}
Q=(\rho, \rho u, \rho v, \rho w, e, \Re)^{T} \\
E=\left[\rho u, \rho u^{2}+p, \rho u v, \rho u w,(e+p) u, \Re u\right]^{T} \\
F=\left[\rho v, \rho v u, \rho v^{2}+p, \rho v w,(e+p) v, \Re v\right]^{T} \\
G=\left[\rho w, \rho w u, \rho w v, \rho w^{2}+p,(e+p) w, \Re w\right]^{T} \\
E_{v}=\frac{M_{\infty}}{\operatorname{Re}_{\infty}}\left(0, \tau_{x x}, \tau_{x y}, \tau_{x z}, E_{v 5}, \frac{-\mu_{t}}{\rho \sigma_{\varepsilon}} \frac{\partial \Re}{\partial x}\right)^{T} \\
F_{v}=\frac{M_{\infty}}{\operatorname{Re}_{\infty}}\left(0, \tau_{x y}, \tau_{y y}, \tau_{y z}, F_{v 5}, \frac{-\mu_{t}}{\rho \sigma_{\varepsilon}} \frac{\partial \Re}{\partial y}\right)^{T} \\
G_{v}=\frac{M_{\infty}}{\operatorname{Re}_{\infty}}\left(0, \tau_{x z}, \tau_{y z}, \tau_{z z}, G_{v 5}, \frac{-\mu_{t}}{\rho \sigma_{\varepsilon}} \frac{\partial \Re}{\partial z}\right)^{T}
\end{gathered}
$$

with

$$
\begin{aligned}
& E_{v 5}=u \tau_{x x}+v \tau_{x y}+w \tau_{x z}-q_{x} \\
& F_{v 5}=u \tau_{x y}+v \tau_{y y}+w \tau_{y z}-q_{y} \\
& G_{v 5}=u \tau_{x z}+v \tau_{y z}+w \tau_{z z}-q_{z}
\end{aligned}
$$

In the preceding equations, $\rho$ is the density; $u, v$, and $w$ are the velocity components; $e$ is the energy per unit volume; and the variable $\Re$ for turbulence model is defined by $k^{2} / \varepsilon$, where $k$ is the turbulent kinetic energy and $\varepsilon$ is the dissipation rate of $k$. The pressure $p$ is related to the dependent variables by the equation of state for a perfect gas:

$$
p=(\gamma-1)\left[e-\rho\left(u^{2}+v^{2}+w^{2}\right) / 2\right]
$$

where $\gamma$ is the ratio of specific heats. The heat flux terms are given by

$$
\begin{gathered}
q_{j}=-\left(K_{l}+K_{t}\right) \frac{\partial T}{\partial x_{j}}, \quad j=1,2,3 \\
K_{l}=\frac{\mu_{l}}{(\gamma-1) P r}, \quad K_{t}=\frac{\mu_{t}}{(\gamma-1) P r_{t}}
\end{gathered}
$$

where $P r=0.72$ and $P r_{t}=0.9$ for air. The viscous stress tensors are obtained from

$$
\begin{gathered}
\tau_{i j}=\left(\mu_{l}+\mu_{t}\right)\left(S_{i j}-\frac{1}{3} \frac{\partial u_{k}}{\partial x_{k}} \delta_{i j}\right) \\
S_{i j}=\frac{1}{2}\left(\frac{\partial u_{i}}{\partial x_{j}}+\frac{\partial u_{j}}{\partial x_{i}}\right)
\end{gathered}
$$

where $i, j=1,2,3$ indicate the three coordinate directions. The molecular viscosity $\mu_{l}$ is calculated by Sutherland's law. The source term is expressed as

$$
\begin{gathered}
H=\left(0,0,0,0,0, H_{6}\right)^{T} \\
H_{6}=\left(C_{\varepsilon 2} f_{2}-C_{\varepsilon 1}\right)(\Re P)^{\frac{1}{2}}+\left(M_{\infty} / R e_{\infty}\right)\left[\mu_{l}+\left(2 \mu_{t} / \sigma_{\varepsilon}\right)\right] \nabla^{2} \Re / \rho
\end{gathered}
$$

where $P$ is the production term of turbulent kinetic energy per unit mass and is given by

$$
P=\frac{M_{\infty}}{R e_{\infty}} \mu_{t}\left[\left(\frac{\partial u_{i}}{\partial x_{j}}+\frac{\partial u_{j}}{\partial x_{i}}\right) \frac{\partial u_{i}}{\partial x_{j}}-\frac{2}{3}\left(\frac{\partial u_{k}}{\partial x_{k}}\right)^{2}\right] / \rho
$$

The quantities $\sigma_{\varepsilon}, C_{\varepsilon 1}$, and $C_{\varepsilon 2}$ are empirical constants in the turbulence model:

$$
\begin{gathered}
1 / \sigma_{\varepsilon}=\left(C_{\varepsilon 2}-C_{\varepsilon 1}\right) C_{\mu}^{\frac{1}{2}} / \kappa^{2} \\
C_{\varepsilon 1}=1.2, \quad C_{\varepsilon 2}=2.0, \quad \kappa=0.41, \quad C_{\mu}=0.09
\end{gathered}
$$

and the eddy viscosity $\mu_{t}$ is given by

$$
\mu_{t}=\left(R e_{\infty} / M_{\infty}\right) C_{\mu} \rho f_{\mu} \Re
$$

Define the turbulent Reynolds number $R e_{T}$ as

$$
R e_{T} \equiv\left(R e_{\infty} / M_{\infty}\right)\left(\rho k^{2} / \varepsilon \mu_{l}\right)=\left(R e_{\infty} / M_{\infty}\right) \rho \Re / \mu_{l}
$$

and the near-wall damping functions $f_{2}$ and $f_{\mu}$ are expressed as

$$
f_{2}=1-0.3 \exp \left(-R e_{T}^{2}\right), \quad f_{\mu}=\frac{1-\exp \left(-A_{\mu} R e_{T}^{2}\right)}{1-\exp \left(-A_{\varepsilon} R e_{T}^{2}\right)}
$$

where

$$
A_{\mu}=4.5 \times 10^{-6}, \quad A_{\varepsilon}=C_{\mu}^{\frac{3}{4}} / 2 \kappa
$$

Note that the near-wall functions $f_{2}$ and $f_{\mu}$ appearing in the preceding formulation are not dependent on wall distance through the parameter $y^{+}$.

The dimensional quantities (denoted by an overbar) are nondimensionalized using freestream conditions (denoted by $\infty$ ) and $\bar{L}$ (the reference length used in the Reynolds number):

$$
\begin{array}{ccc}
x=\bar{x} / \bar{L}, & y=\bar{y} / \bar{L}, \quad z=\bar{z} / \bar{L}, & t=\bar{t} \bar{a}_{\infty} / \bar{L} \\
\rho=\bar{\rho} / \bar{\rho}_{\infty}, & u=\bar{u} / \bar{a}_{\infty}, \quad v=\bar{v} / \bar{a}_{\infty}, & w=\bar{w} / \bar{a}_{\infty} \\
a=\bar{a} / \bar{a}_{\infty}, & p=\bar{p} / \bar{p}_{\infty} \bar{a}_{\infty}^{2}, & T=\bar{T} / \bar{T}_{\infty} \\
\mu_{l}=\bar{\mu}_{l} / \bar{\mu}_{l \infty}, & \mu_{t}=\bar{\mu}_{t} / \bar{\mu}_{l \infty}, & \Re=\bar{\Re} / \bar{L} \bar{a}_{\infty}
\end{array}
$$

where $\bar{a}_{\infty}=\left(\gamma \bar{p}_{\infty} / \bar{\rho}_{\infty}\right)^{1 / 2}$ is the freestream speed of sound.

To allow for the development of a discrete control volume formulation, Eq. (1) is presented in integral form:

$$
\begin{gathered}
\frac{\partial}{\partial t}\left(\frac{1}{V} \int_{V} Q \mathrm{~d} V\right)+\frac{1}{V} \oint_{\Omega}\left(\Im-\Im_{v}\right) \cdot \boldsymbol{n} \mathrm{d} \Omega=H \\
\Im=E \boldsymbol{i}+F \boldsymbol{j}+G \boldsymbol{k}, \quad \Im_{v}=E_{v} \boldsymbol{i}+F_{v} \boldsymbol{j}+G_{v} \boldsymbol{k}
\end{gathered}
$$

where $V$ is the volume of the cell that is bounded by the surface $\Omega$ with the outward unit normal $\boldsymbol{n}$. Here we define the flux at generalized coordinates $(\xi, \eta, \zeta)$ as

$$
\begin{array}{cc}
\hat{E}=\left(\xi_{x} E+\xi_{y} F+\xi_{z} G\right), & \hat{F}=\left(\eta_{x} E+\eta_{y} F+\eta_{z} G\right) \\
\hat{G}=\left(\zeta_{x} E+\zeta_{y} F+\zeta_{z} G\right), & \hat{E}_{v}=\left(\xi_{x} E_{v}+\xi_{y} F_{v}+\xi_{z} G_{v}\right) \\
\hat{F}_{v}=\left(\eta_{x} E_{v}+\eta_{y} F_{v}+\eta_{z} G_{v}\right), & \hat{G}_{v}=\left(\zeta_{x} E_{v}+\zeta_{y} F_{v}+\zeta_{z} G_{v}\right)
\end{array}
$$

where $\boldsymbol{\xi}=\xi_{x} \boldsymbol{i}+\xi_{y} \boldsymbol{j}+\boldsymbol{\xi}_{z} \boldsymbol{k}$ is the surface area vector in $\xi$ direction.

\section{Numerical Method and Boundary Conditions}

\section{Spatial Discretization}

A semidiscrete finite volume method is used to ensure that the final converged solution is independent of the integration procedure and to avoid metric singularity problems. The finite volume method is based on the local flux balance of each mesh cell. The semidiscrete form of Eq. (8) can be written as

$$
\begin{aligned}
\left(\frac{\partial Q}{\partial t}\right)_{i, j, k} & =-\frac{1}{V}\left[\left(\tilde{E}-\tilde{E}_{v}\right)_{i+\frac{1}{2}, j, k}-\left(\tilde{E}-\tilde{E}_{v}\right)_{i-\frac{1}{2}, j, k}\right] \\
- & \frac{1}{V}\left[\left(\tilde{F}-\tilde{F}_{v}\right)_{i, j+\frac{1}{2}, k}-\left(\tilde{F}-\tilde{F}_{v}\right)_{i, j-\frac{1}{2}, k}\right] \\
- & \frac{1}{V}\left[\left(\tilde{G}-\tilde{G}_{v}\right)_{i, j, k+\frac{1}{2}}-\left(\tilde{G}-\tilde{G}_{v}\right)_{i, j, k-\frac{1}{2}}\right]+H_{i, j, k}
\end{aligned}
$$

where $(i, j, k)$ is the control point of finite volume. The spatial differencing adopts WENO schemes ${ }^{11}$ for the inviscid convective fluxes $(\tilde{E}, \tilde{F}, \tilde{G})$ and second-order central differencing for viscous fluxes $\left(\tilde{E}_{v}, \tilde{F}_{v}, \tilde{G}_{v}\right)$. A WENO2 numerical flux at a cell surface $i+\frac{1}{2}$ 
in direction $i$ can be put into the form of a flux limiter method ${ }^{16}$ and is defined by

$$
\tilde{E}_{i+\frac{1}{2}, j, k}=\tilde{E}_{i+\frac{1}{2}, j, k}^{L}+\tilde{E}_{i+\frac{1}{2}, j, k}^{H W}
$$

where $\tilde{E}^{L}$ is the numerical flux of a first-order dissipative entropy satisfying scheme (such as an E-scheme ${ }^{24}$ and $\tilde{E}^{H W}$ is a high-order flux with WENO2 flux limiter. Here the Roe scheme with Harten's entropy fix ${ }^{6}$ is adopted:

$$
\begin{gathered}
\tilde{E}_{i+\frac{1}{2}, j, k}^{L}=\frac{1}{2}\left[\hat{E}\left(Q_{i, j, k}, S_{i+\frac{1}{2}, j, k}\right)+\hat{E}\left(Q_{i+1, j, k}, S_{i+\frac{1}{2}, j, k}\right)\right. \\
\left.\quad-\left(R|\Lambda| R^{-1}\right)_{i+\frac{1}{2}, j, k}\left(Q_{i+1, j, k}-Q_{i, j, k}\right)\right]
\end{gathered}
$$

where $\tilde{E}\left(Q_{i, j, k}, S_{i+1 / 2, j, k}\right)$ is the inviscid flux, the state variables at cell center $(i, j, k)$ and the area vectors at cell face $\left(i+\frac{1}{2}, j, k\right)$ are used. $R$ is the similarity transformation matrix consisting of the right eigenvectors of the Euler system linearized around the Roeaveraged state between $Q_{i+1, j, k}$ and $Q_{i, j, k}$.

$\tilde{E}^{H W}$ is a high-order WENO2 flux, defined as

$$
\begin{aligned}
& \tilde{E}_{i+\frac{1}{2}, j, k}^{H W}=\sum_{s=1}^{6} \tilde{E}_{\left(i+\frac{1}{2}, j, k\right), s}^{H W} \cdot r_{s} \\
& \left.\tilde{E}_{\left(i+\frac{1}{2}, j, k\right.}^{H W}=\left(\omega_{0, s}^{+} / 2\right) \Delta E_{\left(i-\frac{1}{2}, j, k\right), s}^{+}+\left(\omega_{1, s}^{+} / 2\right) \Delta E_{\left(i+\frac{1}{2}, j, k\right.}^{+}\right)_{, s} \\
& \left.\quad-\left(\omega_{0, s}^{-} / 2\right) \Delta E_{\left(i+\frac{1}{2}, j, k\right), s}^{-}-\left(\omega_{1, s}^{-} / 2\right) \Delta E_{\left(i+\frac{3}{2}, j, k\right.}^{-}\right)_{, s}
\end{aligned}
$$

where

$$
\begin{gathered}
\Delta E_{\left(i+\frac{1}{2}, j, k\right), s}^{ \pm}=l_{s} \cdot \Delta E_{i+\frac{1}{2}, j, k}^{ \pm} \\
\Delta E_{i+\frac{1}{2}, j, k}^{+}=\hat{E}\left(Q_{1+1, j, k}, S_{i+\frac{1}{2}, j, k}\right)-\tilde{E}_{i+\frac{1}{2}, j, k}^{L} \\
\Delta E_{i+\frac{1}{2}, j, k}^{-}=\tilde{E}_{i+\frac{1}{2}, j, k}^{L}-\hat{E}\left(Q_{i, j, k}, S_{i+\frac{1}{2}, j, k}\right)
\end{gathered}
$$

The weights $\omega^{ \pm}$are defined by

$$
\omega_{k, s}^{ \pm}=\frac{\alpha_{k, s}^{ \pm}}{\alpha_{0, s}^{ \pm}+\alpha_{1, s}^{ \pm}}, \quad k=0,1
$$

where

$$
\begin{array}{ll}
\alpha_{0, s}^{+}=\frac{1}{3}\left(\varepsilon+I S_{0, s}^{+}\right)^{-2}, & \alpha_{1, s}^{+}=\frac{2}{3}\left(\varepsilon+I S_{1, s}^{+}\right)^{-2} \\
\alpha_{0, s}^{-}=\frac{2}{3}\left(\varepsilon+I S_{0, s}^{-}\right)^{-2}, & \alpha_{1, s}^{-}=\frac{1}{3}\left(\varepsilon+I S_{1, s}^{-}\right)^{-2}
\end{array}
$$

Here $\varepsilon=10^{-30}$ and $I S$ are the smoothness indicators, defined as

$$
\begin{aligned}
& I S_{0, s}^{+}=\left(\Delta E_{\left(i-\frac{1}{2}, j, k\right), s}^{+}\right)^{2}, \quad I S_{1, s}^{+}=\left(\Delta E_{\left(i+\frac{1}{2}, j, k\right), s}^{+}\right)^{2} \\
& I S_{0, s}^{-}=\left(\Delta E_{\left(i+\frac{1}{2}, j, k\right), s}^{-}\right)^{2}, \quad I S_{1, s}^{-}=\left(\Delta E_{\left(i+\frac{3}{2}, j, k\right), s}^{-}\right)^{2}
\end{aligned}
$$

In the preceding equations $r_{s}$ (column vector) and $l_{s}$ (row vector) are the $s$ th right and left eigenvectors of the Jacobian matrices, and they are evaluated using Roe ${ }^{17}$ averages. The $r_{s}$ and $l_{s}$ used in Eqs. (12) and (14), respectively, are evaluated consistently at the $i+\frac{1}{2}$ interface. Note that the WENO2 method just described is only second-order accurate because as a finite difference choice of fluxes (dimension by dimension) is applied to a finite volume setting. However, it is still a genuinely second-order scheme, which does not degenerate to first order at smooth extrema as TVD schemes do.

The high-orderENO flux $\tilde{E}^{H E}$ used for comparison in this work is defined as ${ }^{4}$

$$
\begin{aligned}
& \tilde{E}_{\left(1+\frac{1}{2}, j, k\right), s}^{H E}=\frac{1}{2} m\left(\Delta E_{\left(i-\frac{1}{2}, j, k\right), s}^{+}, \Delta E_{\left(i+\frac{1}{2}, j, k\right), s}^{+}\right) \\
& \quad-\frac{1}{2} m\left(\Delta E_{\left(i+\frac{1}{2}, j, k\right), s}^{-}, \Delta E_{\left(i+\frac{3}{2}, j, k\right), s}^{-}\right)+\varphi_{P}+\varphi_{M}
\end{aligned}
$$

where

$$
m(a, b)=\left\{\begin{array}{lll}
a, & \text { if } & |a| \leq|b| \\
b, & \text { if } & |a|>|b|
\end{array}\right.
$$

$\varphi_{p}=\frac{1}{6}\left\{\begin{array}{rr}2 m\left(\Delta \Delta E_{(i-1, j, k), s}^{+}, \Delta \Delta E_{(i, j, k), s}^{+}\right) & \\ \left.\text {if } \mid \Delta E_{\left(i-\frac{1}{2}, j, k\right.}^{+}\right)_{, s}|\leq| \Delta E_{\left(i+\frac{1}{2}, j, k\right), s}^{+} \mid \\ -m\left(\Delta \Delta E_{(i, j, k), s}^{+}, \Delta \Delta E_{(i+1, j, k), s}^{+}\right) & \text {otherwise }\end{array}\right.$

$$
\varphi_{M}=\frac{1}{6}\left\{\begin{array}{c}
-m\left(\Delta \Delta E_{(i, j, k), s}^{-}, \Delta \Delta E_{(i+1, j, k), s}^{-}\right) \\
\text {if }\left|\Delta E_{\left(i+\frac{1}{2}, j, k\right), s}^{-}\right| \leq\left|\Delta E_{\left(i+\frac{3}{2}, j, k\right), s}^{-}\right| \\
2 m\left(\Delta \Delta E_{(i+1, j, k), s}^{-}, \Delta \Delta E_{(i+2, j, k), s}^{-}\right) \quad \text { otherwise }
\end{array}\right.
$$

The definitions of $\left|\Delta E_{(i+1 / 2, j, k), s}^{ \pm}\right|$are the same as Eqs. (15) and (16) and $\Delta \Delta E_{(i, j, k), s}^{ \pm}$are defined as

$$
\Delta \Delta E_{(i, j, k), s}^{ \pm}=\Delta E_{\left(i+\frac{1}{2}, j, k\right), s}^{ \pm}-\Delta E_{\left(i-\frac{1}{2}, j, k\right), s}^{ \pm}
$$

\section{Time Discretization}

An unfactored implicit scheme can be obtained from a nonlinear implicit scheme by linearizing the flux vectors about the preceding time step and dropping terms of second and higher order:

$$
\begin{aligned}
{[I+} & \left.(\Delta t / V)\left(\delta_{\xi} \hat{A}+\delta_{\eta} \hat{B}+\delta_{\zeta} \hat{C}\right)\right][I-\Delta t \hat{D}] \Delta Q_{i, j, k} \\
& =-(\Delta t / V)\left[\left(\tilde{E}-\tilde{E}_{v}\right)_{i+\frac{1}{2}, j, k}^{n}-\left(\tilde{E}-\tilde{E}_{v}\right)_{i-\frac{1}{2}, j, k}^{n}\right] \\
& -(\Delta t / V)\left[\left(\tilde{F}-\tilde{F}_{v}\right)_{i, j+\frac{1}{2}, k}^{n}-\left(\tilde{F}-\tilde{F}_{v}\right)_{i, j-\frac{1}{2}, k}^{n}\right] \\
& -(\Delta t / V)\left[\left(\tilde{G}-\tilde{G}_{v}\right)_{i, j, k+\frac{1}{2}}^{n}-\left(\tilde{G}-\tilde{G}_{v}\right)_{i, j, k-\frac{1}{2}}^{n}\right] \\
& +\Delta t H_{i, j, k} \equiv \operatorname{RHS}
\end{aligned}
$$

where $I$ is the identity matrix; $n$ is the time level; $\delta_{\xi}, \delta_{\eta}$, and $\delta_{\zeta}$ are the differenceoperators, $\hat{A}, \hat{B}$, and $\hat{C}$ are the Jacobianmatrices of inviscid fluxes; $\hat{D}=\partial H / \partial Q ; \Delta Q=Q^{n+1}-Q^{n}$ and is the increment of conservative variables; and RHS is right-hand side. Note that the viscous terms are treated explicitly, and the turbulent source functions are treated implicitly. Because the production term is positive, its linearization is not possible; however, there is a strong coupling between the flowfield, turbulent viscosity, and the production term. The stiffness caused by the production term can be reduced by using the following pseudolinearization ${ }^{25}$ :

$$
\frac{\partial H_{6}}{\partial \Re}=-\frac{|P|}{0.1 \Re}
$$

The matrix inversion resulting from the source-term linearization is performed before the spatial sweeps:

$$
\begin{gathered}
{\left[I+(\Delta t / V)\left(\delta_{\xi} \hat{A}+\delta_{\eta} \hat{B}+\delta_{\zeta} \hat{C}\right)\right] \Delta Q_{i, j, k}} \\
=\mathrm{RHS} /[I-\Delta t \hat{D}] \equiv \mathrm{RHS}^{*}
\end{gathered}
$$

The LU-SGS implicit factorization scheme of Yoon and Jameson ${ }^{20}$ for Eq. (28) can be derived by combining the advantages of LU factorization and SGS relaxation. The LU-SGS scheme can be written as

$$
L D^{-1} U \Delta Q=\text { RHS }^{*}
$$


where

$$
\begin{gathered}
L=I+(\Delta t / V)\left(\delta_{\xi}^{-} \hat{A}^{+}+\delta_{\eta}^{-} \hat{B}^{+}+\delta_{\zeta}^{-} \hat{C}^{+}-\hat{A}^{-}-\hat{B}^{-}-\hat{C}^{-}\right) \\
D=I+(\Delta t / V)\left(\hat{A}^{+}-\hat{A}^{-}+\hat{B}^{+}-\hat{B}^{-}+\hat{C}^{+}-\hat{C}^{-}\right) \\
U=I+(\Delta t / V)\left(\delta_{\xi}^{+} \hat{A}^{-}+\delta_{\eta}^{+} \hat{B}^{-}+\delta_{\zeta}^{+} \hat{C}^{-}+\hat{A}^{+}+\hat{B}^{+}+\hat{C}^{+}\right)
\end{gathered}
$$

where $\delta_{\xi}^{-}, \delta_{\eta}^{-}$, and $\delta_{\zeta}^{-}$are backward difference operators and $\delta_{\xi}^{+}, \delta_{\eta}^{+}$, and $\delta_{\zeta}^{+}$are forward difference operators. Split Jacobian matrices of the flux vectors are constructed so that the eigenvalues of + matrices are nonnegative and those of - matrices are nonpositive, that is,

$$
\hat{A}^{ \pm}=R_{\xi} \Lambda_{\xi}^{ \pm} R_{\xi}^{-1}, \quad \hat{B}^{ \pm}=R_{\eta} \Lambda_{\eta}^{ \pm} R_{\eta}^{-1}, \quad \hat{C}^{ \pm}=R_{\zeta} \Lambda_{\zeta}^{ \pm} R_{\zeta}^{-1}
$$

where $R_{\xi}$ and $R_{\xi}^{-1}$ are similarity transformation matrices of the eigenvectors of $\hat{A}$. The Jacobian matrices $\hat{A}_{i+1 / 2, j, k}^{ \pm}$are computed using the Roe-averaged ${ }^{17}$ state between $Q_{i+1, j, k}$ and $Q_{i, j, k}$ and the area vectors at cell face $\left(i+\frac{1}{2}, j, k\right)$. Equation (29) can be inverted in three steps:

$$
\begin{gathered}
\Delta Q^{*}=L^{-1} \mathrm{RHS}^{*} \\
\Delta Q^{* *}=D Q^{*} \\
\Delta Q=U^{-1} Q^{* *}
\end{gathered}
$$

Note that the present implicit algorithm (LU-SGS) is completely vectorizable on $i+j+k=$ const oblique plane of sweep.

\section{Boundary Conditions}

The mean flow and turbulent transport equations presented in preceding sections represent an initia-boundary-valueproblem. To solve these equations, it is necessary to impose initial and boundary conditions. A uniform flowfield is chosen as the initial conditions for the mean flow equations. A uniform value of $\Re \approx 10^{-4}\left(v_{t} \approx 1000\right)$ is set as the initial guess.

The boundary conditions of mean flow are set as follows: 1) No-slip boundary conditions for velocities are adopted on the solid surface, which is assumed to be an adiabatic wall. 2) The density and pressure on the wall are set to be equal to the values of the node points next to the wall. This gives first-order accuracy at the wall. 3 ) In the far field, a locally one-dimensional characteristic type of boundary condition is used. For the turbulent transport equation, a zeroth-order extrapolation is used to specify conditions at the far field. The value of $\Re$ is set to zero at the solid wall.

\section{Results and Discussion}

Presented here are the results of two different two-dimensional turbulent flows and one three-dimensional turbulent flow computations to illustrate and test the codes. The two-dimensional cases are the transonic turbulent flows over NACA 0012 and RAE 2822 airfoil. The three-dimensional case is transonic turbulent flow over an ONERA M6 wing. We compare our results with available experimental data and other computational results for each case.

\section{Flow over NACA 0012 Airfoil}

The first result is the transonic flow over a NACA 0012 airfoil at freestream condition $M_{\infty}=0.799, \alpha=2.26 \mathrm{deg}$, and $R e_{c}=9 \times 10^{6}$. The angle of attack ( $2.26 \mathrm{deg})$ used in the computation is obtained from the measured angle of attack $(2.86 \mathrm{deg})$ using a linear windtunnel-wall correction procedure. For this transonic flowfield, a shock wave exists on the airfoil upper surface at about $x / c=0.5$, which is strong enough to cause significant boundary-layer separation. This case represents a severe test for all solution methods in terms of both numerical algorithm as well as turbulence models The calculation is performed on an O-type grid. The grid system (Fig. 1) around the airfoil is $241 \times 45$, with 113 points on upper surface, 113 points on lower surface and 17 points on blunt trailing edge, that is, base region, The mesh extends from the airfoil surface to a circle of the far-field boundary located approximately 50 chord lengths from the body and the first grid line at a distance of $7 \times 10^{-6}$ chord length off the wall, which resulted in a min $y^{+}<1.5$ over the entire grid; here $y^{+}<u_{\tau} y / v$, where $u_{\tau}$ is the friction velocity.

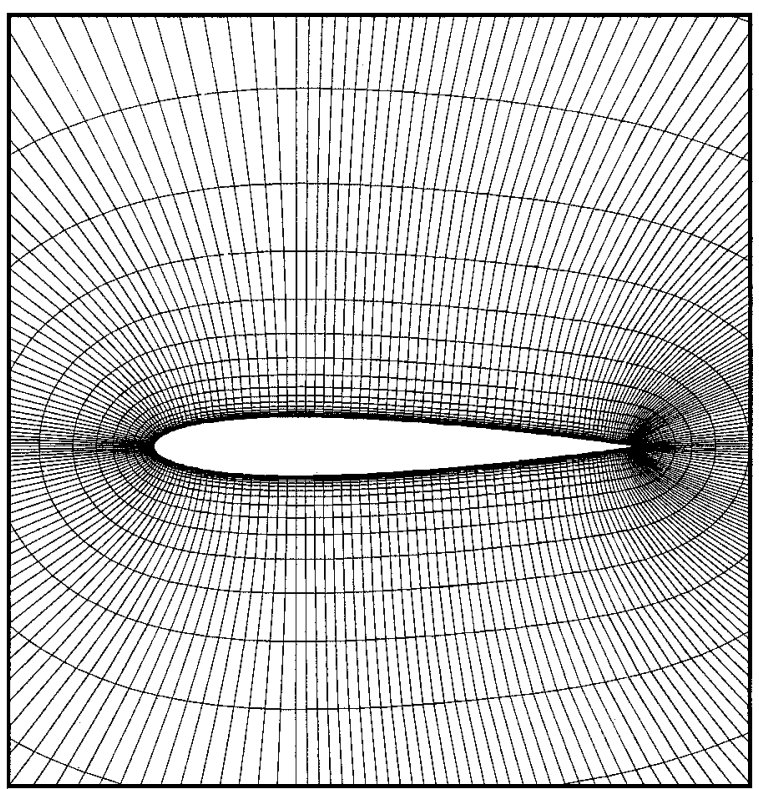

Fig. 1 O-type grid $241 \times 45$ for NACA 0012 airfoil.

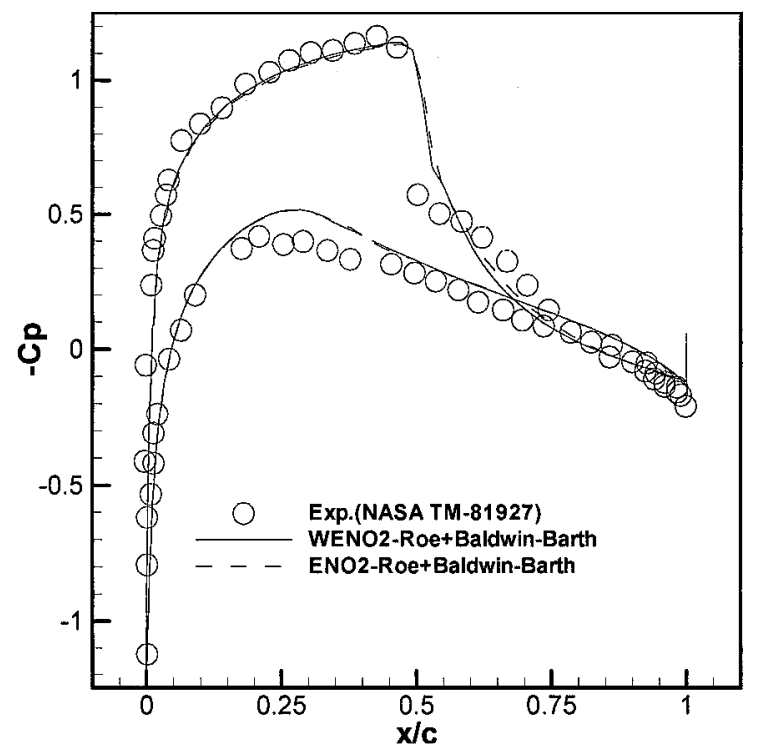

Fig. 2 NACA 0012 airfoil surface pressure distribution at $M_{\infty}=0.799$, $\alpha=2.26 \mathrm{deg}$, and $R e_{c}=9 \times 10^{6}$; comparison of WENO-Roe and ENORoe schemes.

The solutions were calculated using WENO2-Roe and ENO2Roe scheme, where Roe refers to the first-order flux Roe scheme. ${ }^{17}$ The calculations presented here have been computed using local time stepping at constant Courant numbers of 3.0. Figure 2 shows the comparison of surface pressure distributions with the experimental data. ${ }^{26}$ The computed result is in good agreement with experimental data except for a slight discrepancy in the postshockposition and the magnitude of lower surface pressure. Computed lift and drag coefficients of WENO2-Roe scheme are $C_{L}=0.335$ and $C_{D}=0.0325$. The experimental values of lift and drag coefficients given by Harris are $C_{L}=0.391$ and $C_{D}=0.033$. Figure 3 shows the contours of constant Mach numbers, all of the flow features including the front leading edge structure, the supersonic pocket, and shock separation are clearly resolved. Figure 4 shows the convergence history. After the residuals have decayed for three orders of magnitude, the convergence of ENO2 scheme is leveling off, whereas monotone convergence can be achieved with WENO2 schemes.

\section{Flow over RAE 2822 Airfoil}

The next computation is for the transonic flow over an RAE 2822 airfoil that has been tested extensively by Cook et al. ${ }^{27}$ This airfoil is a supercritical airfoil with a significant amount of aft camber. 


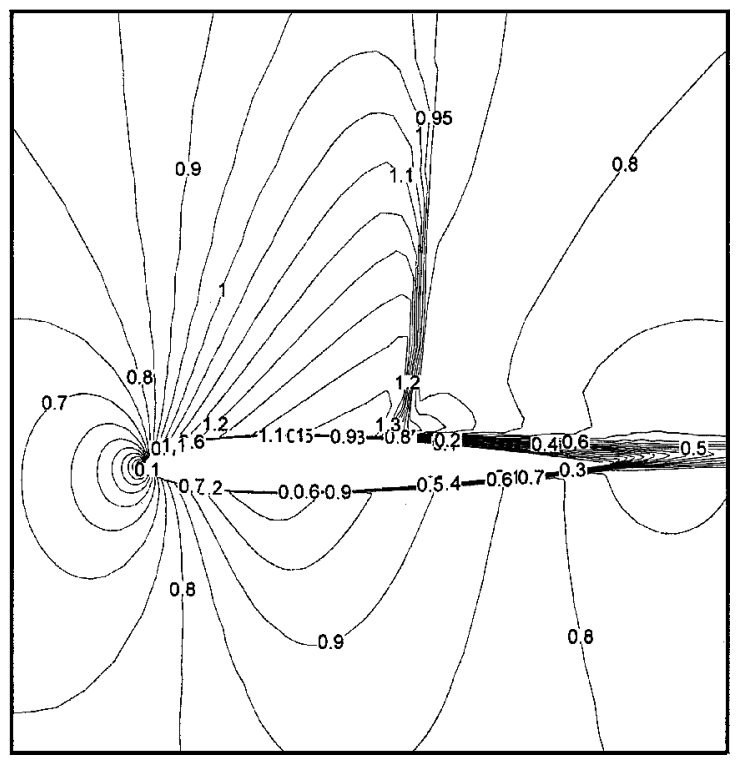

Fig. 3 Mach number contours for NACA 0012 airfoil at $M_{\infty}=0.799$, $\alpha=2.26 \mathrm{deg}$, and $\operatorname{Re}_{c}=9 \times 10^{6}$; WENO2-Roe scheme.

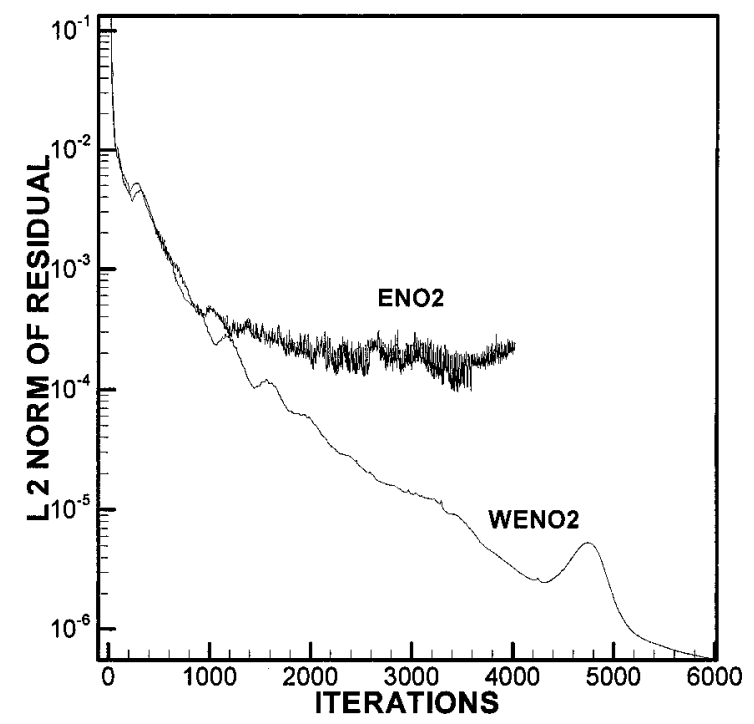

Fig. 4 Convergence history for NACA 0012 airfoil at $M_{\infty}=0.799$, $\alpha=2.26 \mathrm{deg}$, and $R_{\mathrm{c}}=9 \times 10^{6}$.

Solutions were obtained of this case on four O-type meshes consisting of $241 \times 45,181 \times 45,121 \times 45$, and $177 \times 45$ grid points in the streamwise and normal directions, respectively. The trailing edges of the first three grid systems are blunt and that of the last grid system is sharp. The fine-grid system (Fig. 5) is similar to that used in the NACA 0012 airfoil test case.

The result is for the transonic flow over an RAE 2822 airfoil at freestreamcondition $M_{\infty}=0.725$, angle of attack $\alpha=2.92 \mathrm{deg}$, and reference Reynolds number based on airfoil chord, $R e_{c}=6,5 \times 10^{6}$ corresponding to case 6 in the experimental study of Cook et al. ${ }^{27}$ Because of the presence of wall interference effects in the experiment, the corrected flow conditions with $M_{\infty}=0.731$ and $\alpha=2.51$ as suggested by Tatsumi et al. ${ }^{28}$ are used. This flow involves a strong shock wave at $x / c=0.55$ on the upper surface. The lift coefficient in this case depends strongly on the predicted shock location. This requires a good resolution of the shock wave. Jiang et al. ${ }^{29}$ have computed this problem using convective upwind split pressure scheme with Baldwin-Barth one-equation turbulence model. ${ }^{18}$

In Fig. 6, the computed pressure coefficient distributions of the fine grid system of WENO2 and ENO2 schemes are shown and compared with the experiment. The present results are in close agreement with experimental data in all aspects. Figure 7 shows

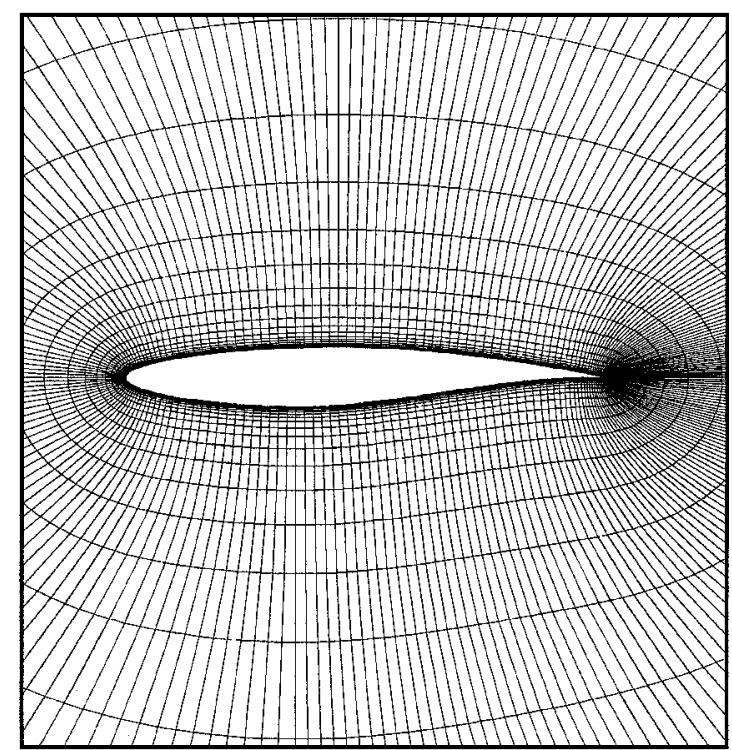

Fig. 5 O-type grid $241 \times 45$ for RAE 2822 airfoil.

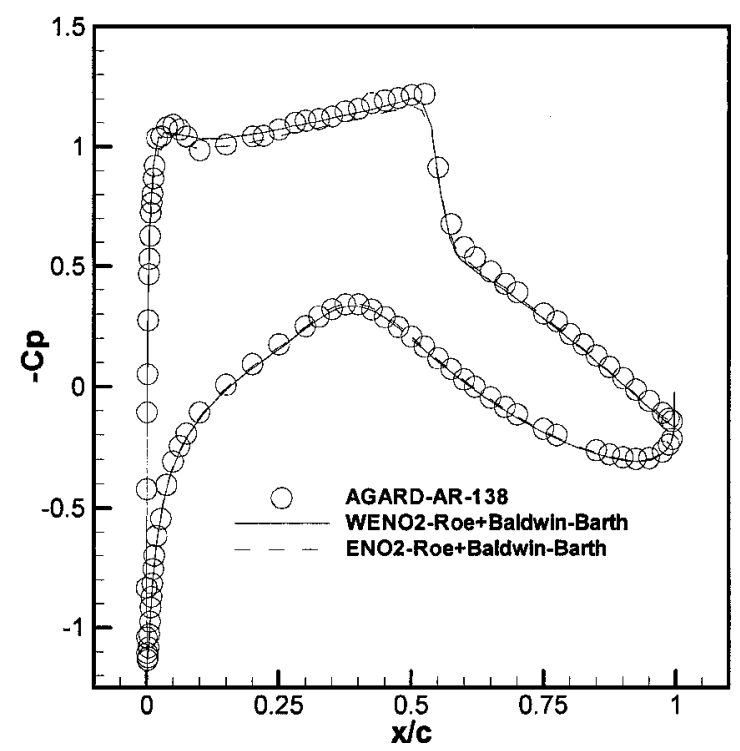

Fig. 6 RAE 2822 airfoil surface pressure distribution at $M_{\infty}=0.731$, $\alpha=2.51 \mathrm{deg}$, and $R_{c}=6.5 \times 10^{6}$; comparison of WENO2 and ENO2 schemes.

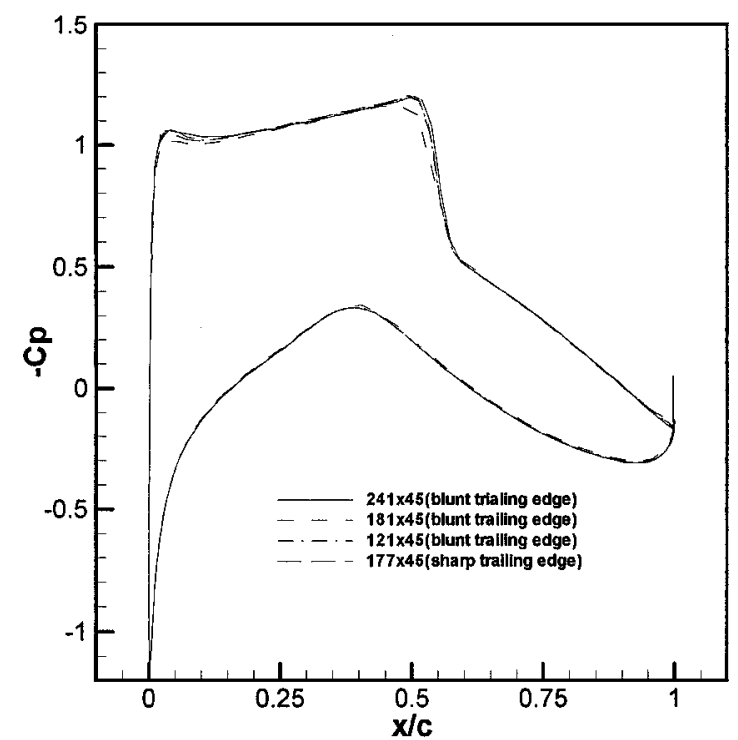

Fig. 7 RAE 2822 airfoil surface pressure distribution at $M_{\infty}=0.731$, $\alpha=2.51 \mathrm{deg}$, and $R e_{c}=6.5 \times 10^{6} ;$ comparison of different grid systems. 
Table 1 Lift and drag coefficients for RAE 2822 airfoil at $M_{\infty}=0.725, \alpha=2.92 \mathrm{deg}$, and $R e_{c}=6.5 \times 10^{6}$

\begin{tabular}{lcccc}
\hline \hline Scheme & Grid & Trailing edge & $C_{L}$ & $C_{D}$ \\
\hline AGARD & & & 0.743 & 0.0127 \\
Jiang et al. $^{29}$ & $384 \times 64$ & & 0.702 & 0.0088 \\
WENO2 & $241 \times 45$ & Blunt & 0.740 & 0.0151 \\
WENO2 & $181 \times 45$ & Blunt & 0.737 & 0.0148 \\
WENO2 & $121 \times 45$ & Blunt & 0.718 & 0.0138 \\
WENO2 & $177 \times 45$ & Sharp & 0.738 & 0.0148 \\
ENO2 & $241 \times 45$ & Blunt & 0.719 & 0.0149 \\
\hline \hline
\end{tabular}

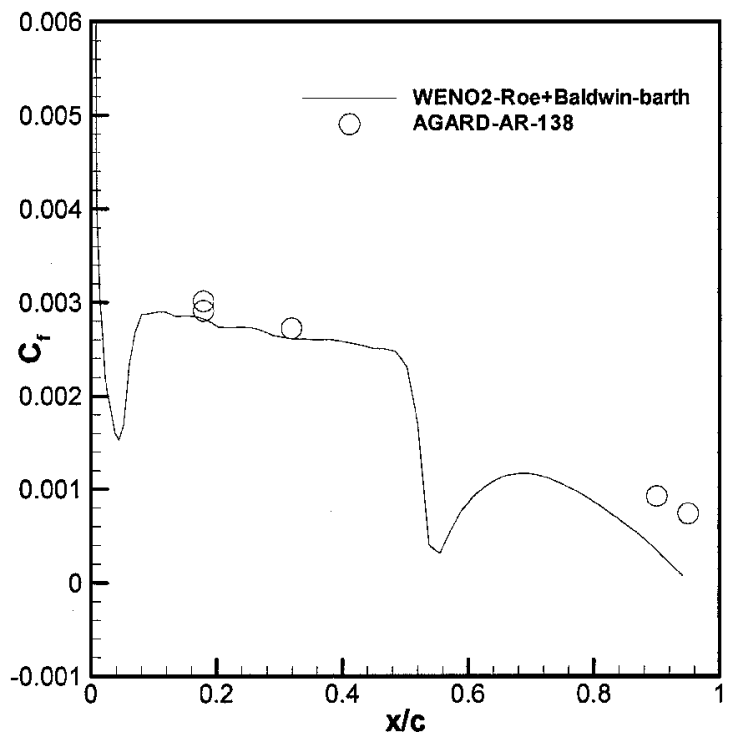

Fig. 8 Skin-friction distribution of the upper surface of RAE 2822 airfoil at $M_{\infty}=0.731, \alpha=2.51 \mathrm{deg}$, and $R e_{c}=6.5 \times 10^{6}$; WENO2-Roe scheme.

the results of the WENO2 scheme on different grid systems. A comparison of the calculated results of the experimental data and the Jiang et al. ${ }^{29}$ results is shown in Table 1 . Notice that the flow is grid resolved and that the sharp trailing edge produces almost the same solutions as that obtained with a blunt trailing edge. Computed skin-friction distribution from the upper surface of the RAE 2822 airfoil for the case just presented is compared with experimental data in Fig. 8. The skin-friction values are referred to the boundarylayer edge dynamic pressure. Generally, the computed results are in good agreement with experiment, with exceptions near the leading edge, where the skin-friction quantity is difficult to define, and near the trailing edge. The computed skin-friction coefficients by Jiang et al. ${ }^{29}$ do not agree well with available experimental data. Figure 9 shows the contours of constant Mach numbers. Figure 10 shows the convergence history. Again, the WENO scheme gives a good convergence rate. Figure 11 shows the convergence of lift and drag of the fine grid system of the WENO2-Roe scheme.

Our computed results for both the NACA 0012 and RAE 2822 airfoils are consistent with those given in the extensive compendium of results by Holst. ${ }^{30}$

\section{Three-Dimensional Transonic Flow over ONERA M6 Wing}

The result of a three-dimensional case is the transonic flow over an ONERA M6 wing at a $M_{\infty}=0.8395$ and with 3.06-deg angle of attack and reference Reynolds number $R e_{c}=2.6 \times 10^{6}$. The ONERA M6 wing is a symmetric airfoil section with a sweep angle of $30 \mathrm{deg}$. The wing is tapered with a taper ratio of 0.56 and has an aspect ratio of 3.8. Extensive wind-tunnel test data exist for the ONERA M6 wing, in particular, the pressure data for transonic flow conditions. ${ }^{31}$ Takakura et al. ${ }^{32}$ have computed this problem using the Harten-Yee TVD scheme (see Ref. 5) together with the Jones-Launder $k-\varepsilon$ model.

Our calculation is performed on an O-O-type grid system, containing $160 \times 25 \times 44$ cells in the wraparound, spanwise, and bodynormal directions, respectively. The outer boundaries were extended to a mesh system that extends to 30 chord lengths in all directions.

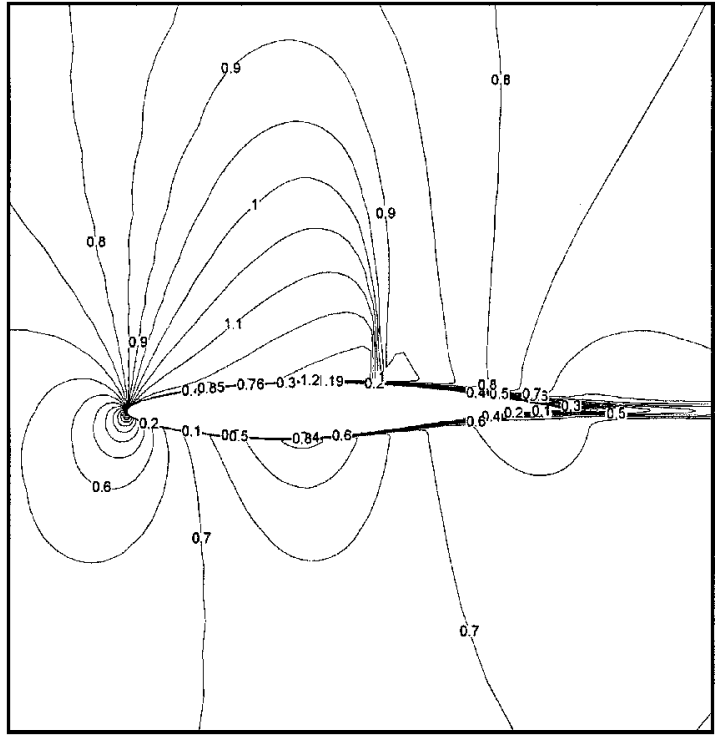

Fig. 9 Mach number contours for RAE 2822 airfoil at $M_{\infty}=\mathbf{0 . 7 3 1}$, $\alpha=2.51 \mathrm{deg}$, and $R e_{c}=6.5 \times 10^{6}$; WENO2-Roe scheme.

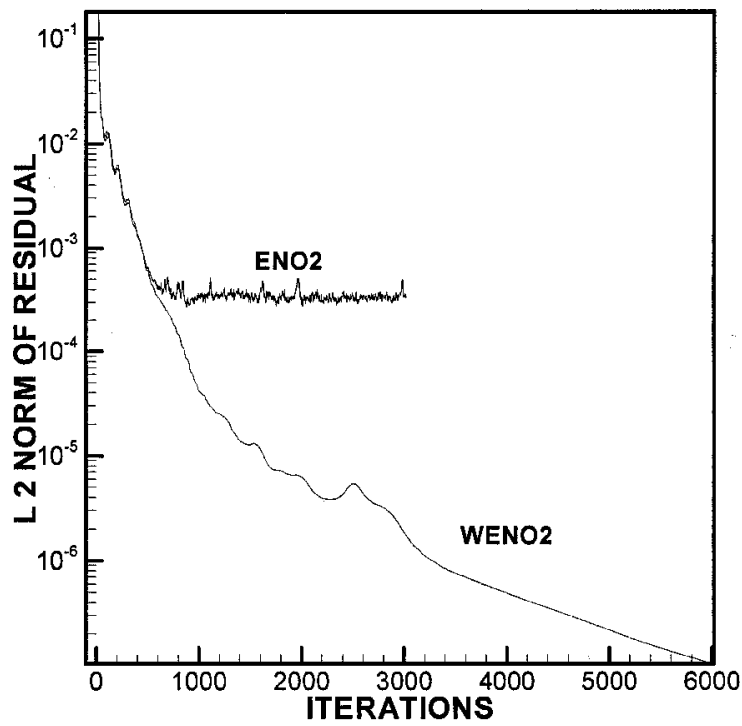

Fig. 10 Convergence history for RAE 2822 airfoil at $M_{\infty}=\mathbf{0 . 7 3 1}$, $\alpha=2.51 \mathrm{deg}$, and $R e_{c}=6.5 \times 10^{6}$.

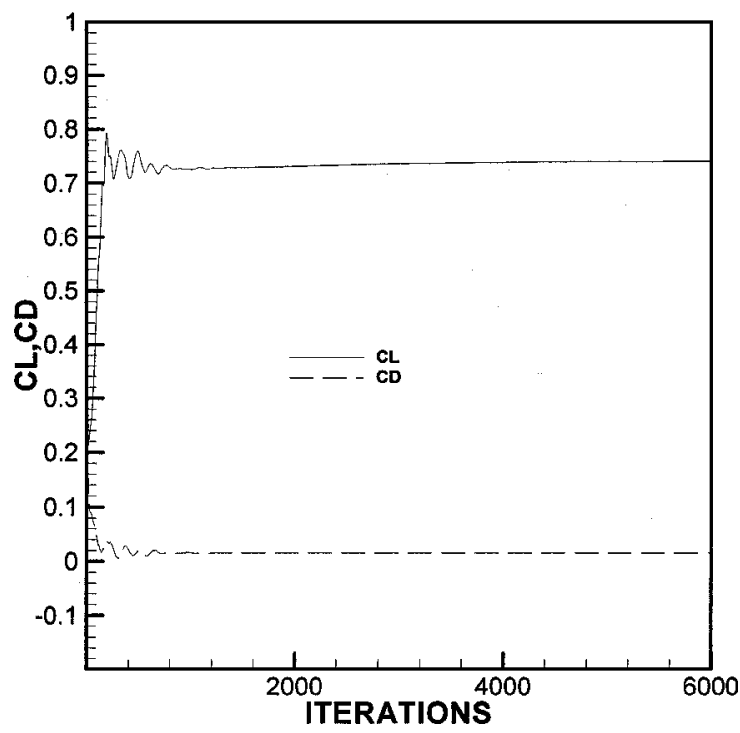

Fig. 11 Convergence of lift and drag coefficients of WENO2-Roe scheme for RAE 2822 airfoil at $M_{\infty}=0.731, \alpha=2.51 \mathrm{deg}$, and $R e_{c}=$ $6.5 \times 10^{6}$. 


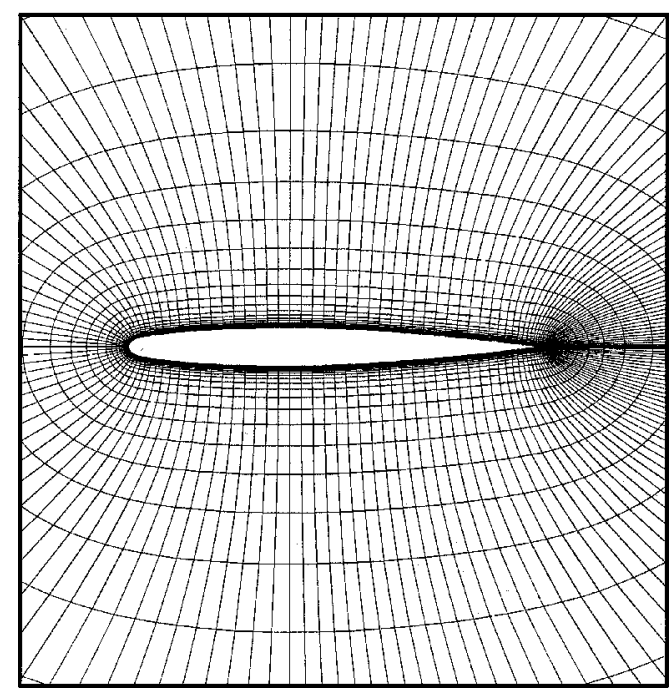

Fig. 12 O-type grid $161 \times 45$ for ONERA M6 wing at symmetrical $(j=1)$ plane.

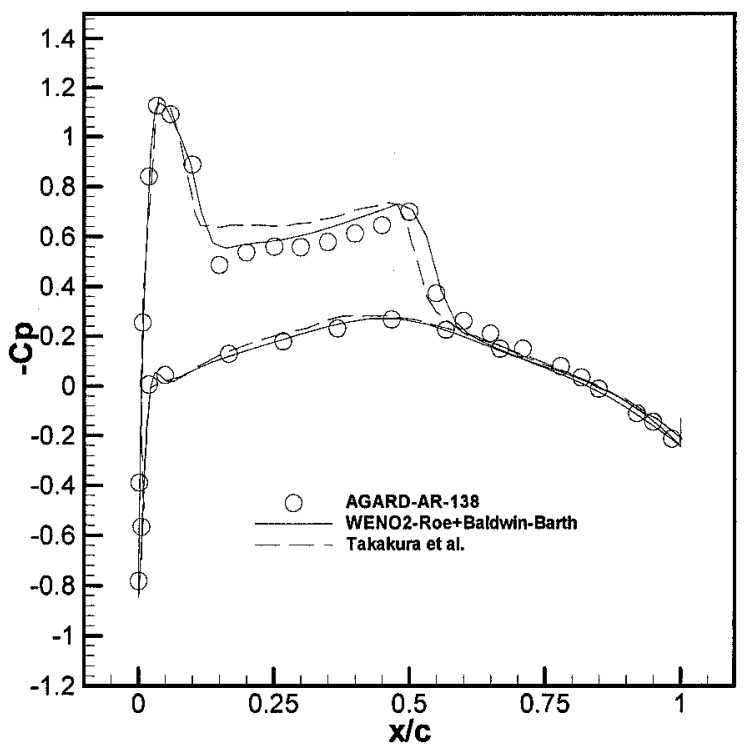

a) $y / b=0.44$

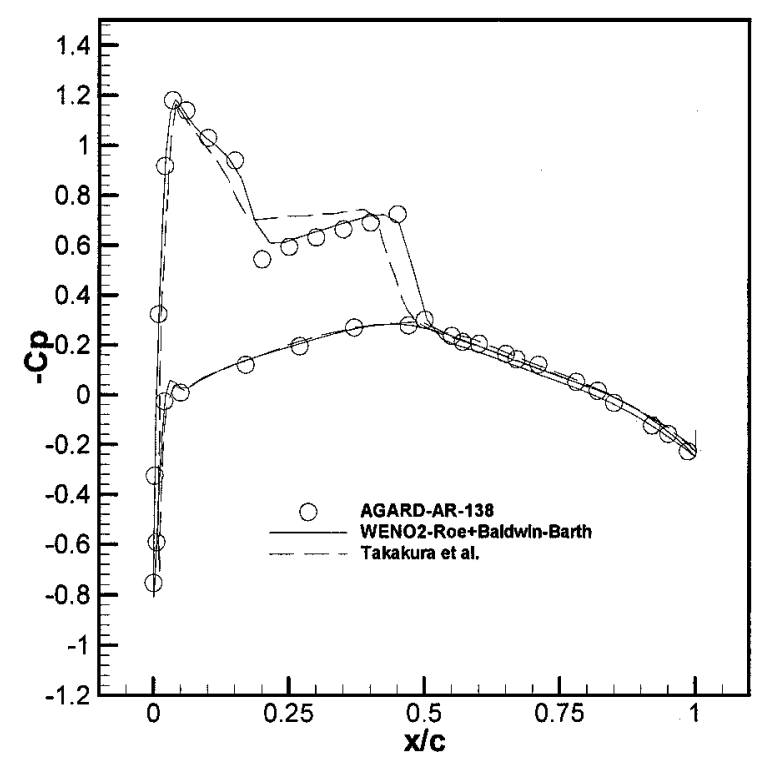

b) $y / b=0.65$
The first grid line is at a distance of $10^{-5}$ chord length off the wall, which resulted in a min $y^{+}<2.0$ over the entire grid. The grid system was generated by letting the $j=1$ plane (Fig. 12) be the plane of grid points on the upper wing surface at the root section. The $j$ planes were then distributed nonlinearly along the upper surface to $j=21$ at the upper surface tip. Planes 22-26 were then rotated in a circular arc to model the wing tip. This wraparound wing tip allows the modeling of the wing tip as it existed in the wind-tunnel model.

The solutions were calculated using WENO2-Roe schemes at local Courant-Friedrichs-Lewy number 20.0. In Fig. 13, we show the surface pressure coefficients of the present scheme as compared with experimental data ${ }^{31}$ and the other calculations by Takakura et al. ${ }^{32}$ (The number of grid points is $191 \times 33 \times 24$.) It is shown that our numerical results are in good agreement with the experimental data and are more accurate than the results of Takakuraet al. in terms of both shock location and strength. This test case was at transonic condition, which results in a double-shocksconfiguration, which is evident in Figs. 13a-13c. Finally, Fig. 13d shows the shocks having coalesced to form one at the 0.25 chord position, and this shock is by far the strongest shock of all of those observed in Fig. 13. The configuration obviously results in the lambda double-shock

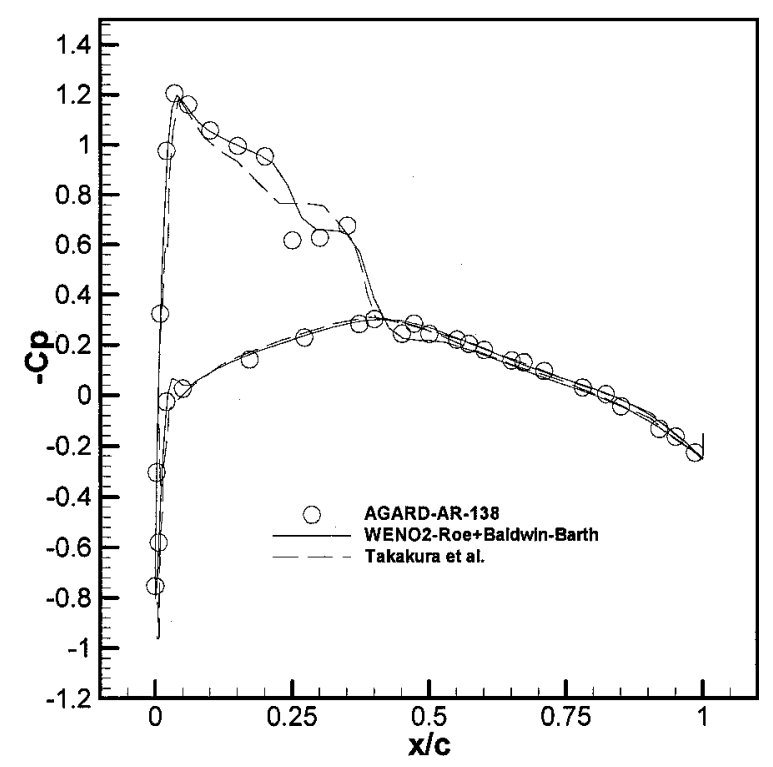

c) $y / b=0.80$

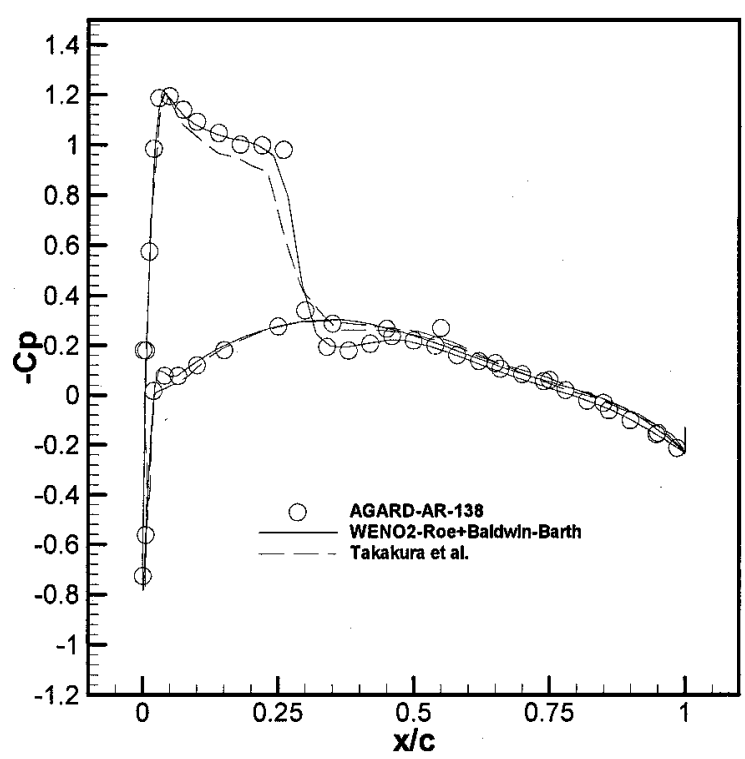

d) $y / b=0.90$

Fig. 13 Steady pressure distributions for ONERA M6 wing at $M_{\infty}=0.8395, \alpha=3.06 \mathrm{deg}$, and $R e_{c}=2.6 \times 10^{6}$. 


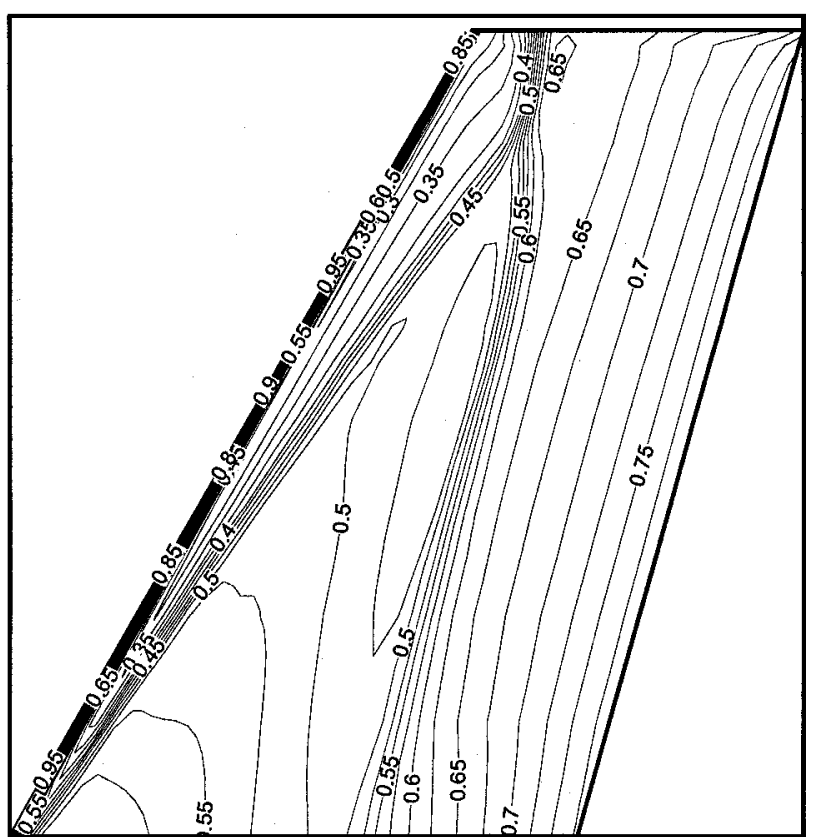

Fig. 14 Upper surface pressure contours for ONERA M6 wing at $M_{\infty}=0.8395, \alpha=3.06 \mathrm{deg}$, and $R e_{c}=2.6 \times 10^{6}$.

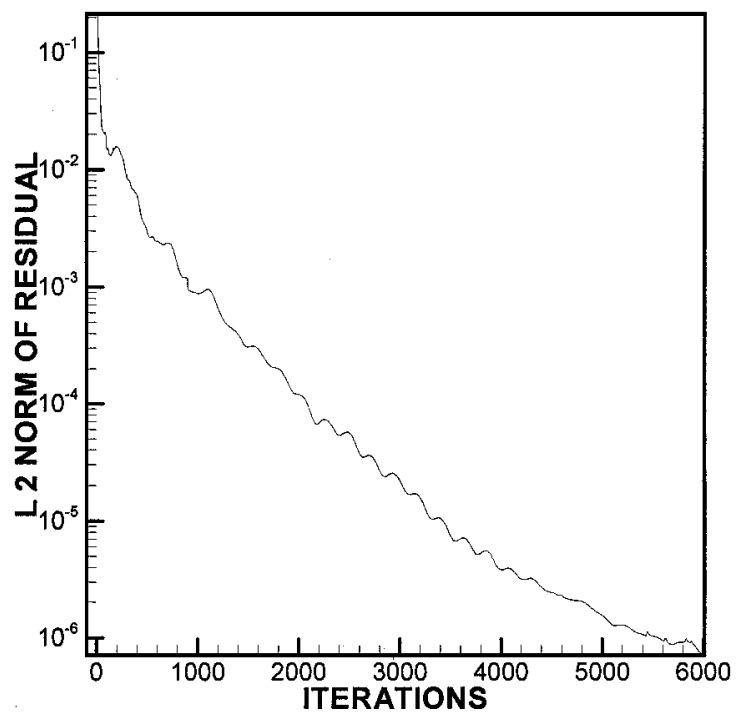

Fig. 15 Convergence history for ONERA M6 wing at $M_{\infty}=\mathbf{0 . 8 3 9 5}$, $\alpha=3.06 \mathrm{deg}$, and $R e_{c}=2.6 \times 10^{6}$; WENO2-Roe scheme.

pattern for transonic conditions on a swept wing. Figure 14 shows the pressure contours along the upper surface and the double-shocks pattern coalescing into a single shock at the tip can be observed. Figure 15 shows the convergence history.

\section{Conclusions}

High-resolution numerical codes for solving the two- and threedimensional compressible Navier-Stokes equations with pointwise version of Baldwin-Barth ${ }^{18}$ one-equation turbulence model have been developed. The present method adopts a numerical flux in flux limiter form for the WENO spatial operator for convective flux that allows for a flexiblility to implement various first-order entropy satisfying dissipative schemes. The integration of equations is via the implicit LU-SGS algorithm. Applications to turbulent transonic flows over NACA 0012 and RAE 2822 airfoils and three-dimensional turbulent flow over an ONERA M6 wing have been carried out to validate and illustrate the codes. The use of a WENO spatial operator for the inviscid fluxes not only enhances the accuracy but also improves the convergencerate for steady-state computation as compared with using the ENO counterpart. It is found that, for all cases computed, the solutions of the present algorithms are in good agreement with the experimental data and other available computational results.

\section{Acknowledgments}

This work was done under the auspices of National Science Council, Taiwan, through Grant NSC-87-2212-E002-071. We thank Horng-Tsair Lee, Herng Lin, and Shih-Chang Yang of the ChungShan Institute of Science and Technology for many useful discussions. The authors wish to thank the reviewers for many constructive comments and suggestions.

\section{References}

${ }^{1}$ Harten, A., Engquist, B., Osher, S., and Chakravarthy, S., "Uniformly High-Order Accurate Nonoscillatory Scheme, III," Journal of Computational Physics, Vol. 71, 1987, pp. 231-303.

${ }^{2}$ Shu, C.-W., and Osher, S., "Efficient Implementation of Nonoscillatory Shock Capturing Schemes," Journal of Computational Physics, Vol. 77, 1988, pp. 439-471.

${ }^{3}$ Shu, C.-W., and Osher, S., "Efficient Implementation of Nonoscillatory Shock Capturing Schemes, II," Journal of Computational Physics, Vol. 83, 1989, pp. 32-78.

${ }^{4}$ Shu, C.-W., "Essentially Non-Oscillatory and Weighted Essentially NonOscillatory Schemes for Hyperbolic Conservation Laws," Advanced Numerical Approximation of Nonlinear Hyperbolic Equations, edited by A. Quarteroni, Vol. 1697, Lecture Notes in Mathematics, Springer, 1998, pp. 325-432.

${ }^{5}$ Yee, H.-C., and Harten, A., "Implicit TVD Schemes for Hyperbolic Conservation Laws in Curvilinear Coordinates," AIAA Journal, Vol. 25, No. 2 , 1987, pp. 266-274.

${ }^{6}$ Harten, A., "A High Resolution Scheme for the Computation of Weak Solutions of Hyperbolic Conservation Laws," Journal of Computational Physics, Vol. 49, 1983, pp. 357-393.

${ }^{7}$ Rogerson, A. M., and Meiburg, E., "A Numerical Study of the Convergence Properties of ENO Schemes," Journal of Scientific Computing, Vol. 5, No. 2, 1990, pp. 151-167.

${ }^{8}$ Shu, C. W., "Numerical Experiments on the Accuracy of ENO and Modified ENO Schemes," Journal of Scientific Computing, Vol. 5, No. 2, 1990, pp. 127-149.

${ }^{9}$ Casper, J., Shu, C. W., and Atkins, H., "Comparison of Two Formulations for High-Order Accurate Essentially Nonoscillatory Schemes," AIAA Journal, Vol. 32, No. 10, 1994, pp. 1970-1977.

${ }^{10}$ Liu, X.-D., Osher, S., and Chan, T., "Weighted Essentially Nonoscillatory Schemes," Journal of Computational Physics, Vol. 115, 1994, pp. 200-212.

${ }^{11}$ Jiang, G.-S., and Shu, C.-W., "Efficient Implementation of Weighted ENO Schemes," Journal of Computational Physics, Vol. 126, 1996, pp. 202-228.

${ }^{12}$ Atkins, H., "High-Order ENO Methods for the Unsteady Compressible Navier-Stokes Equations," AIAA Paper 91-1557, 1991.

${ }^{13}$ Chen, Y.-N., Yang, S.-C., and Yang, J.-Y., "Implicit Weighted ENO Schemes for the Incompressible Navier-Stokes Equations," International Journal for Numerical Methods in Fluids, Vol. 31, 1999, pp. 747-765.

${ }^{14}$ Yang, J.-Y., Yang, S.-C., Chen, Y.-N., and Hsu, C.-A., "Weighted ENO Schemes for the Three-Dimensional Incompressible Navier-Stokes Equations," Journal of Computational Physics, Vol. 146, 1998, pp. 464-487.

${ }^{15}$ Chorin, A. J., "A Numerical Method for Solving Incompressible Viscous Flow Problems," Journal of ComputationalPhysics, Vol. 2, 1967, pp. 12-26.

${ }^{16}$ LeVeque, R. J., Numerical Method for Conservation Laws, Birkhäuser Verlag, 1990, p. 176.

${ }^{17}$ Roe, P., "Approximate Riemann Solvers, Parameter Vectors and Difference Schemes," Journal of Computational Physics, Vol. 43, 1981, pp. 357-372.

${ }^{18}$ Baldwin, B. S., and Barth, T. J., "A One-Equation Turbulence Transport Model for High Reynolds Number Wall-Bounded Flows," AIAA Paper 91-0610, 1991.

${ }^{19}$ Goldberg, U.-C., and Ramakrishnan, S.-V., "A Pointwise Version of Baldwin-Barth Turbulence Model," Computational Fluid Dynamics, Vol. 1, 1993, pp. 321-338.

${ }^{20}$ Yoon, S., and Jameson, A., "Lower-Upper Symmetric-Gauss-Siedel Method for the Euler and Navier-Stokes Equaions," AIAA Journal, Vol. 26, 1988,pp. 1025, 1026.

${ }^{21}$ Yoon, S., and Kwak, D., "Three-Dimensional Incompressible NavierStokes Solver Using Lower-Upper Symmetric-Gauss-Seidel Algorithm," AIAA Journal, Vol. 29, 1991, pp. 874, 875.

${ }^{22}$ Yoon, S., and Kwak, D., "Implicit Navier-Stokes Solver for ThreeDimensional Compressible Flow," AIAA Journal, Vol. 30, 1992, pp. 26532658. 
${ }^{23}$ Yoon, S., and Kwak, D., "Multigrid Convergence of an Implicit Symmetric Relaxation Scheme," AIAA Journal, Vol. 32, No. 5, 1994, pp. 950955.

${ }^{24}$ Osher, S., "Riemann Solver, the Entropy Condition, and Difference Approximations," SIAM Journal on Numerical Analysis, Vol. 21, 1984, pp. 984-995.

${ }^{25}$ Siikonen, T., "An Application of Roe's Flux-Difference Spliting for $\kappa-\varepsilon$ Turbulence Model," International Journal for Numerical Methods in Fluids, Vol. 21, 1995, pp. 1017-1039.

${ }^{26}$ Harris, C.-D., "Two-Dimensional Aerodynamic Characteristics of the NACA0012 Airfoil in the Langley 8-Foot Transonic Pressure Tunnel," NASA TM-81927, 1981.

${ }^{27}$ Cook, P.-H., McDonald, M.-A., and Firmin, M. C. P., "Airfoil RAE2822 Pressure Distributions and Boundary Layer and Wake Measurement," AR138-A6, AGARD, 1979.
${ }^{28}$ Tatsumi, S., Martinelli, L., and Jameson, A., "A New High Resolution Scheme for Compressible Flows Past Airfoil," AIAA Paper 95-0466, 1995.

${ }^{29}$ Jiang, Y. T., Damodaran, M., and Lee, K. H., "High Resolution Finite Volume Calculation of Turbulent Transonic Flow Past Airfoils," AIAA Paper 96-2377, 1996

${ }^{30}$ Holst, T. L., "Viscous Transonic Airfoil Workshop Compendium of Results," Journal of Aircraft, Vol. 25, 1988, pp. 1073-1087.

${ }^{31}$ Schimit, V., and Charpin, F., "Pressure Distributions on the ONERA M6 Wing at Transonic Mach Numbers," AR-138-B1, AGARD, 1979.

${ }^{32}$ Takarura, Y., Ogawa, S., and Ishiguro, T., "Turbulence Models for 3D Transonic Viscous Flows," AIAA Paper 89-1952, 1989.

P. Givi

Associate Editor 